\title{
The relationship between self-rated health, physical function and activity capacity in adult burn patients 6 and 12 months after injury.
}

\author{
Enblom S, OT MSc, Guné G E, OT, Sundin E, PT, Aspling N, PT \\ Burn Center, Uppsala University Hospital, Uppsala, Sweden
}

\begin{abstract}
Aim
To examine the relationship between self-rated health, physical function and capacity in performing activities among adults with burns.
\end{abstract}

\section{Background}

Approximately 38,000 people in Sweden seek medical care due to burns each year. About 400 of them need specialized care at a Burn center.

Physical function and daily activities are often affected after a burn injury. As a consequence this might also affect the experience of health related QoL. We expected that burn victims with reduced physical function and/or capacity in performing activities would have decreased self-rated health.

\section{Results}

There was a significant correlation at 6 months post burn between self-rated health (EQ-5D VAS) and

\section{- activity performance (DASH, Check your health Activity) \\ - muscle function (IMF) \\ - the patients' subjective scar assessment (POSAS)}

The significant correlations at $\mathbf{6}$ months were not found at 12 months except for self-rated activity (Check your health Activity). There were no significant correlation between self-rated health (EQ-5D) and TBSA \% as was expected.

\begin{tabular}{|c|c|c|c|c|c|c|}
\hline Correlations to EQ-5D & \multicolumn{3}{|c|}{6 months } & \multicolumn{3}{|c|}{12 months } \\
\hline & $\mathrm{n}$ & $\mathrm{p}$ & $\mathrm{r}$ & $\mathrm{n}$ & $\mathrm{p}$ & $\mathrm{r}$ \\
\hline $\begin{array}{l}\text { Check your health } \\
\text { (Burns) Activity }{ }^{1}\end{array}$ & 29 & $0,000 * *$ & 0,711 & 32 & $0,000 * *$ & 0,760 \\
\hline $\mathrm{DASH}^{1}$ & 28 & $0,001 * *$ & $-0,613$ & 27 & 0,135 & $-0,295$ \\
\hline Function assessment ${ }^{1}$ & 24 & 0,318 & 0,213 & 25 & 0,899 & 0,027 \\
\hline IMF total ${ }^{1}$ & 16 & $0,002 * *$ & $-0,708$ & 17 & 0,751 & $-0,083$ \\
\hline POSAS $^{1}$ & 38 & 0,000 ** & $-0,552$ & 37 & 0,087 & $-0,285$ \\
\hline TBSA $^{2}{ }^{2}$ & 43 & 0,841 & 0,032 & 37 & 0,270 & $-0,186$ \\
\hline
\end{tabular}

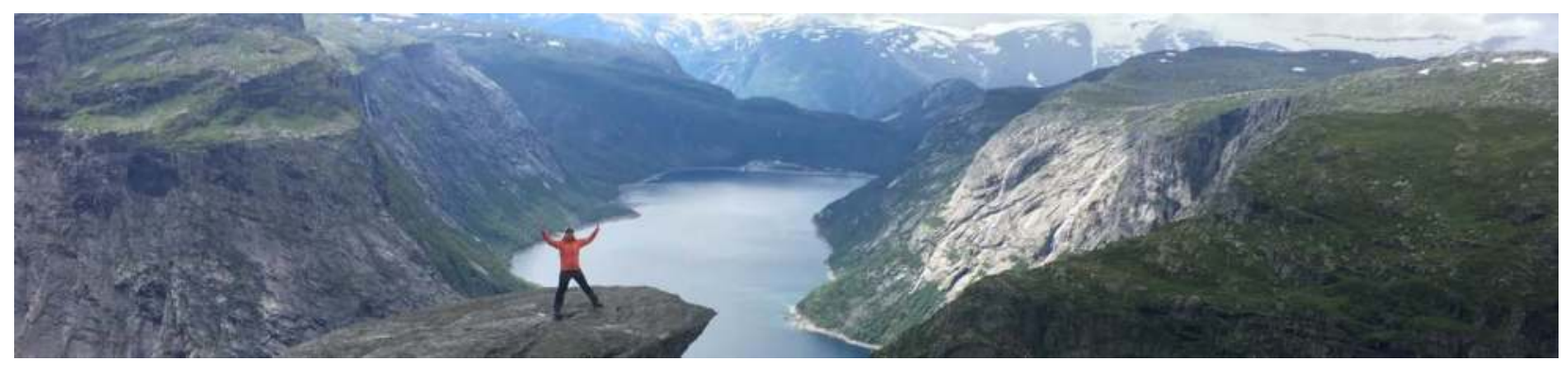

\section{Method}

Adult burn patients that come for follow-up at the Uppsala University Hospital Burn Center 6 and 12 months post-burn were asked to participate and were assessed by treating OT and PT.

50 consecutive patients were included from November 2015 to February 2017, 35 men (70\%) and 15 women aged 18 to 80 years (mean=44,5) with a burn injury of 0,25 to 83,5 TBSA \% (mean=13,5). Length of stay varied from 1 to 113 days $(m=17,4)$.

\begin{tabular}{|c|c|c|c|c|}
\hline nge) & & 6 months & & 12 months \\
\hline & $\mathrm{n}$ & median (range) SD & $\mathrm{n}$ & median (range) SD \\
\hline $\begin{array}{l}\text { Check your health - } \\
\text { QoL }(0-100)\end{array}$ & 41 & $80,0(30-100) 20,0$ & 37 & $80,0(40-100) 16,3$ \\
\hline $\begin{array}{l}\text { Check your health - } \\
\text { Activity }(0-100)\end{array}$ & 30 & $82,5(40-100) 19,5$ & 32 & $85,0(50-100) 15,0$ \\
\hline DASH $(0-100)$ & 30 & $10,8(0-68,75) 19,9$ & 27 & $5,8(0-54,2) 17,8$ \\
\hline EQ-5D VAS $(0-100)$ & 44 & $80,0(20-100) 19,2$ & 38 & $80,0(30-100) 15,8$ \\
\hline $\begin{array}{l}\text { Function assessment } \\
(10-60)\end{array}$ & 26 & 58,5 (29-60) 6,8 & 25 & $60,0(33-60) 5,8$ \\
\hline IMF total $(0-40)$ & 18 & $0,0(0-21) 6,6$ & 17 & $0,0(0-23) 6,6$ \\
\hline $\begin{array}{l}\text { POSAS Patient, } \\
\text { Overall (1-10) }\end{array}$ & 38 & $6,0(1-10) 2,6$ & 37 & $7,0(2-10) 2,2$ \\
\hline \multicolumn{5}{|c|}{$\begin{array}{l}\text { Assessments: } \\
\text {-Disabilities of the Arm, Shoulder and Hand Questionnaire } \\
\text { (DASH) } \\
\text {-EQ-5D Health Questionnaire } \\
\text { - The Patient and Observer Scar Assessment Scale (POSAS) } \\
\text {-IMF (Index of Muscle Function) - lower extremities } \\
\text { - Function assessment - upper extremities } \\
\text { - Check your health (Burns) (Wikblad et al. 2013) }\end{array}$} \\
\hline
\end{tabular}

\section{Conclusion}

The level of physical function affects self-rated health early post burn. Gradually other factors are more likely to affect the health assessment. However, small subgroups make the results uncertain. 